\title{
Adiabaticity of the ramping process of an ac dipole
}

\author{
R. Tomás \\ Brookhaven National Laboratory, Upton, New York 11973, USA
}

(Received 1 July 2004; published 1 February 2005)

\begin{abstract}
ac dipoles in accelerators are used to excite coherent betatron oscillations at a drive frequency close to the tune. If the excitation amplitude is slowly increased to the desired value and slowly decreased back to zero there is no significant emittance growth. The aim of this article is to study the adiabaticity of the ramping process of an ac dipole as a function of the different parameters involved.
\end{abstract}

DOI: 10.1103/PhysRevSTAB.8.024401

PACS numbers: 41.85.-p, 29.27.-a

\section{INTRODUCTION}

ac dipoles were introduced in accelerators to overcome depolarization due to intrinsic spin resonances [1,2] and as a powerful tool for machine diagnostics. More recently, ac dipoles were proposed to be used for measuring the nonlinear content of an accelerator [3,4], and presently different experiments are being performed to test this technique [5-10]. There is experimental evidence of the need of slowly ramping the ac dipole excitation amplitude in order to ensure the adiabaticity of the process. The lack of adiabaticity implies a transfer of energy to the natural betatron motion of the beam, which results in an increase of the transverse beam sizes. It is crucial to know what the parameters are that affect the adiabaticity of the ramping process of the ac dipole to obtain its best possible performance. This article derives analytical expressions of the particle motion during the ramping process and studies its adiabaticity. Transverse nonlinearities and chromaticity are taken into account separately. Collective effects such as space charge are neglected.

To analytically assess the adiabaticity of a ramping process the following scenario is considered. The beam initially is centered in the transverse phase space and does not perform any oscillations. After the ramp takes place the forced oscillation is ideally removed (or ideally ramped down). The remaining free beam oscillation plus the emittance blowup represent the total transfer of energy. Therefore the parameter that accounts for the nonadiabaticity has to be the emittance blowup plus the square of the centroid oscillation, i.e.,

$$
\boldsymbol{\epsilon}_{x f}-\boldsymbol{\epsilon}_{x 0}+\left\langle x_{f}\right\rangle^{2}=\left\langle x_{f}^{2}\right\rangle-\boldsymbol{\epsilon}_{x 0},
$$

where $\epsilon_{x f}$ and $\epsilon_{x 0}$ are the final and initial emittances defined as $\epsilon_{x}=\left\langle x^{2}\right\rangle-\langle x\rangle^{2}$. The brackets \langle\rangle mean the average over the particle distribution and the turn number $T$. The term $\left\langle x_{f}^{2}\right\rangle$ is computed considering only the betatron oscillation of tune $Q_{x}$, then $x_{f}$ has the form $x_{f}(T)=$ $|z| \cos \left(2 \pi Q_{x} T+\phi\right)$, and therefore

$$
\left\langle x_{f}^{2}\right\rangle=\left\langle|z|^{2} \cos ^{2}\left(2 \pi Q_{x} T+\phi\right)\right\rangle=\frac{1}{2}\left\langle|z|^{2}\right\rangle .
$$

The nonadiabaticity of a ramp is then characterized by the following expression,

$$
\frac{1}{2}\left\langle|z|^{2}\right\rangle-\epsilon_{x 0}
$$

where $|z|$ is the amplitude of the natural beam oscillations after the ac dipole ramp.

\section{THE LINEAR CASE}

Consider that the excitation amplitude of an ac dipole is not ramped linearly but is increased by a certain quantity after every $N$ turns, as depicted in Fig. 1. The regions where the excitation remains constant are labeled by an integer which, in general, will be represented by the letter $n$. The analytical expression describing the turn-by-turn motion in the region $n$ is expressed as [3]

$$
\begin{aligned}
\hat{x}(T)-i \hat{p}_{x}(T)= & z^{(n)} e^{i 2 \pi Q_{x} T}+\delta_{-}^{(n)} e^{i 2 \pi Q_{D} T} \\
& -\delta_{+}^{(n)} e^{-i 2 \pi Q_{D} T},
\end{aligned}
$$

where $\hat{x}$ and $\hat{p}_{x}$ are the linearly normalized coordinates, $T$ is the turn number, $z^{(n)}$ is a complex quantity containing the amplitude and phase of the oscillation with the natural frequency in the region $n$, and $\delta_{-}^{(n)}$ and $\delta_{+}^{(n)}$ are given by

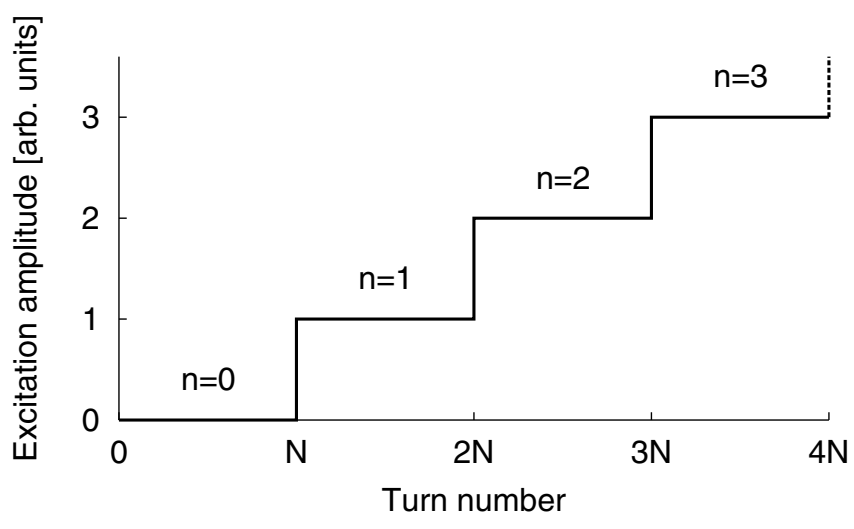

FIG. 1. Excitation amplitude of the ac dipole versus turn number. 


$$
\delta_{ \pm}^{(n)}=\sqrt{\beta_{D}} \frac{B L^{(n)}}{\left(B_{0} \rho\right)} \frac{e^{ \pm i\left(\pi Q_{ \pm}-\psi_{0}\right)}}{4 \sin \left(\pi Q_{ \pm}\right)},
$$

where $\beta_{D}$ is the betatron function at the ac dipole, $B L^{(n)}$ is the integrated magnetic field of the ac dipole at the $n$th step, $B_{0} \rho$ is the magnetic rigidity, $\psi_{0}$ is the initial phase of the ac dipole, and $Q_{ \pm}=Q_{D} \pm Q_{x}$. Notice that close to the resonance $Q_{D}=Q_{x}$ the quantity $\delta_{-}^{(n)}$ is much larger than $\delta_{+}^{(n)}$. The amplitude of $z^{(n)}$ serves as an estimate for the nonadiabaticity of the ramp up to the $n$th step. In order to compute $z^{(n)}$ the continuity of the particle position is imposed between the regions of constant excitation amplitude. Defining $\delta_{ \pm}=\delta_{ \pm}^{(n+1)}-\delta_{ \pm}^{(n)}$, the continuity condition between the regions $n$ and $n+1$ is expressed as

$$
\begin{aligned}
z^{(n)} e^{i 2 \pi Q_{x} N(n+1)}+\delta_{-}^{(n)} e^{i 2 \pi Q_{D} N(n+1)}-\delta_{+}^{(n)} e^{-i 2 \pi Q_{D} N(n+1)}= & z^{(n+1)} e^{i 2 \pi Q_{x} N(n+1)}+\left(\delta_{-}^{(n)}+\delta_{-}\right) e^{i 2 \pi Q_{D} N(n+1)}-\left(\delta_{+}^{(n)}\right. \\
& \left.+\delta_{+}\right) e^{-i 2 \pi Q_{D} N(n+1)}
\end{aligned}
$$

Simplifying this expression the following relation is obtained:

$$
z^{(n+1)}=z^{(n)}-\delta_{-} e^{i 2 \pi Q_{-} N(n+1)}+\delta_{+} e^{-i 2 \pi Q_{+} N(n+1)} .
$$

By expressing $z^{(n)}$ as a function of $z^{(n-1)}$ and doing this successively, the previous equation takes the form

$$
z^{(n+1)}=z^{(0)}-\sum_{j=0}^{n} \delta_{-} e^{i 2 \pi Q_{-} N(j+1)}+\sum_{j=0}^{n} \delta_{+} e^{-i 2 \pi Q_{+} N(j+1)} .
$$

By computing the summations over $j, z^{(n)}$ is given by

$$
\begin{aligned}
z^{(n)}= & z^{(0)}-\delta_{-} \frac{\sin \left(\pi Q_{-} N n\right)}{\sin \left(\pi Q_{-} N\right)} e^{i \pi Q_{-} N(n+1)} \\
& +\delta_{+} \frac{\sin \left(\pi Q_{+} N n\right)}{\sin \left(\pi Q_{+} N\right)} e^{-i \pi Q_{+} N(n+1)} .
\end{aligned}
$$

This expression is valid for any width of the steps $N$ and provides the amplitude and phase of the natural motion in the region $n$. From this equation it is easy to show that, for given top excitation amplitude and number of turns of operation, the minimum $\left|z^{(n)}\right|$ is given by taking $N=1$ $\left[z^{(0)}=0\right.$ is assumed]. This is the linear ramp. In order to give a simple expression for the nonadiabaticity, Eq. (2), of a linear ramp, the term with $\delta_{-}$is assumed to be the most relevant in Eq. (8). The amplitude of the oscillation with the natural frequency is given by

$$
\left|z^{(n)}\right|=\delta_{-}^{(n)} \frac{\sin \left(\pi Q_{-} n\right)}{n \sin \left(\pi Q_{-}\right)},
$$

where $\delta_{-}$has been expressed as $\delta_{-}^{(n)} / n$ since a linear ramp has been assumed. Note that the evaluation of Eq. (2) from Eq. (9) is trivial since we are in the linear case and $z^{(n)}$ does not depend on any phase space variable. From Eq. (9) the lengths of the ramp $n$ that transfer no energy to the natural oscillation are those fulfilling $n Q_{-}=p, p$ being any integer. For example, having $Q_{-}=0.02$, a 50 turns ramp is enough to ensure adiabaticity in a linear machine. This is illustrated by the curve with the label "zero detuning" of Fig. 3, where $\left|z^{(n)}\right| /\left|\delta_{-}^{(n)}\right|$ at the last step has been plotted versus the number of steps for a given excitation amplitude at the flattop, i.e., for constant $\left|\delta_{-}^{(n)}\right|$.

\section{THE NONLINEAR CASE}

In this section the beam motion during the ramp of the ac dipole in the presence of nonlinearities is derived in a similar way as in the previous section. The general solution of the turn-by-turn motion in the presence of a constant ac excitation and nonlinearities is given by [4]

$$
\hat{x}-i \hat{p}_{x}=\zeta_{x}^{-}-2 i \sum_{j k l m} j \frac{h_{j k l m}}{1-e^{-i 2 \pi Q_{x}} R_{x, y} R_{\tau}} \zeta_{x}^{+^{(j-1)}} \zeta_{x}^{-k} \zeta_{y}^{+^{l}} \zeta_{y}^{-m} \text {, }
$$

where $h_{j k l m}$ are the Hamiltonian terms, $R_{x, y}$ is the linear one turn map acting on the betatron phases $\psi_{x, y}, R_{\tau}$ is the one turn map acting on the timelike variable $\tau$ of the excitation, and

$$
\begin{aligned}
\zeta_{x}^{ \pm}= & \sqrt{2 I_{x}} e^{\mp i\left(\psi_{x}+\psi_{x 0}\right)}+\left|\delta_{x-}\right| e^{\mp i\left(2 \pi Q_{D} \tau-\eta_{x-}\right)} \\
& -\left|\delta_{x+}\right| e^{ \pm i\left(2 \pi Q_{D} \tau+\eta_{x+}\right)} \\
\zeta_{y}^{ \pm}= & \sqrt{2 I_{y}} e^{\mp i\left(\psi_{y}+\psi_{y 0}\right)}
\end{aligned}
$$

where $I_{x}$ is the nonlinear invariant and, for simplicity, the equations hold only at the location of the ac dipole. The time evolution of these expressions is due to $\psi_{x, y}$ and $\tau$,

$$
\begin{gathered}
\psi_{x, y}(T)=2 \pi Q_{x, y}\left(I_{x}, I_{y},\left|\delta_{-}\right|,\left|\delta_{+}\right|\right) T, \\
\tau(T)=T,
\end{gathered}
$$

where it has been explicitly stated that the machine tunes are functions of the amplitudes of all the oscillations. The same ramp as in the previous section is assumed. By virtue of Eq. (10) the continuity of $\hat{x}-i \hat{p}_{x}$ between consecutive steps implies the continuity of $\zeta_{x}^{ \pm}$, expressed as

$$
\begin{aligned}
\sqrt{2 I_{x}^{(n+1)}} e^{i\left(2 \pi Q_{x}^{(n+1)} T+\psi_{x 0}^{(n+1)}\right)}= & \sqrt{2 I_{x}^{(n)}} e^{i\left(2 \pi Q_{x}^{(n)} T+\psi_{x 0}^{(n)}\right)} \\
& -\delta_{-} e^{i 2 \pi Q_{D} T}+\delta_{+} e^{-i 2 \pi Q_{D} T} .
\end{aligned}
$$

The only difference between this equation and Eq. (5) is that the tunes of different steps are different since the oscillation amplitudes are different. This effect makes it very difficult to obtain analytical expressions describing 


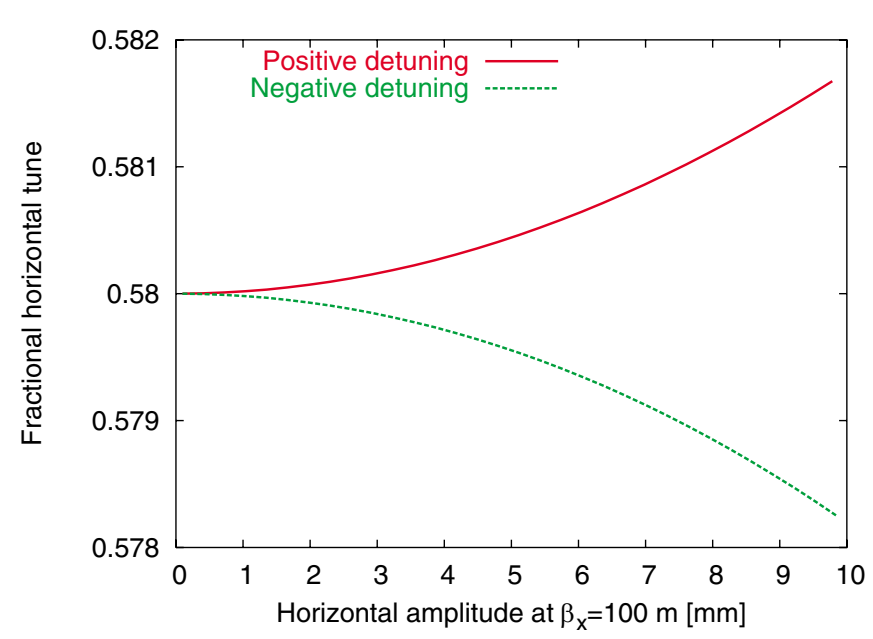

FIG. 2. (Color) Amplitude detunings used in the simulations.

the adiabaticity of the ramping process and numerical studies are more suitable. To illustrate the effect of amplitude detuning, single particle and multiparticle simulations have been performed using a lattice of the CERN Super Proton Synchrotron (SPS) containing positive, negative, and zero amplitude detuning. One of the octupole families devoted to the Landau damping has been used to introduce an absolute amplitude detuning 4 times larger than that usually measured in the SPS [11]. The actual amplitude detuning used in the simulations is shown in Fig. 2. The natural tune of the lattice is 26.58 and the tune of the forced oscillation is 26.60 . In the case with positive amplitude detuning $Q_{-}$decreases as the excitation amplitude increases. The excitation amplitudes are chosen to obtain a variation of $Q_{-}$of approximately $\pm 10 \%$ in the cases of negative and positive amplitude detuning, respectively. The tracking code SIXTRACK [12] was used to simulate a single particle under the influence of an ac dipole for different ramp lengths and a constant excitation amplitude during the flattop. In Fig. 3 the amplitude of the natural tune oscillation normalized to the amplitude of the forced oscillation in the flattop is plotted versus the number of turns of the ramp for the mentioned three cases. The case with zero amplitude detuning is in complete accordance with Eq. (9). The cases with detuning have a similar behavior than the previous one with slightly different $Q_{-}$ and without ever dropping to zero.

The adiabaticity of the ramp for a particle distribution is addressed in the following. Ideally this particle distribution should be a two dimensional Gaussian over the horizontal phase space; however, due to CPU time limitations the study had to be restricted to one dimension (particles with the same amplitude). The single particle case presented above can be regarded as describing the adiabaticity of particles close to the core of the beam. Now particles having an amplitude equal to a typical beam size are considered. To this aim 20 particles with an oscillation amplitude of $3 \mathrm{~mm}$ at a $\beta_{x}=100 \mathrm{~m}$ (this corresponds to a

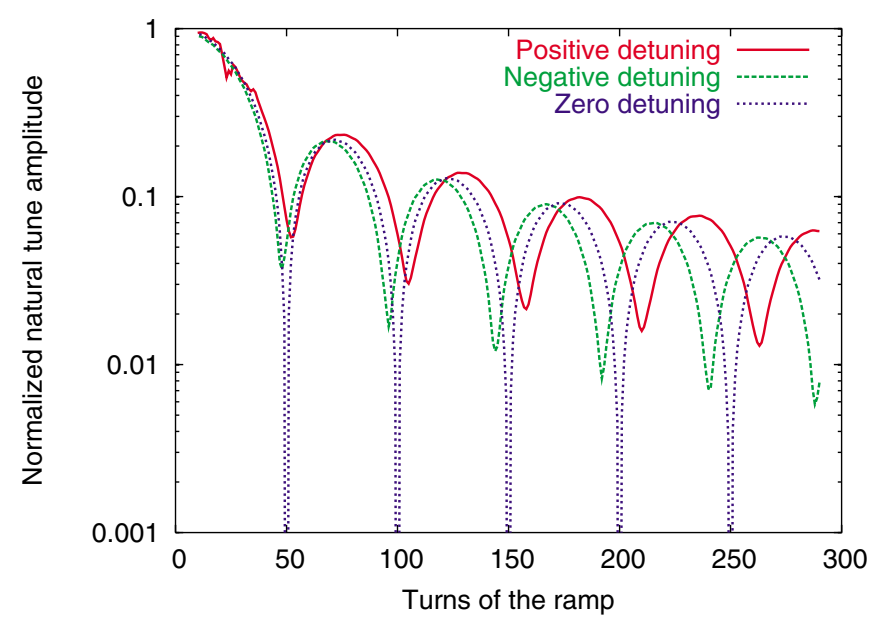

FIG. 3. (Color) Amplitude of the natural oscillation of the particle normalized to the amplitude of the forced oscillation in the flattop versus the number of turns used to ramp the excitation for negative, positive, and zero amplitude detuning.

typical SPS beam size) and evenly distributed in phase have been tracked for different ramp lengths. Given the regularity of the phase space 20 particles were found sufficient to provide a good average. In order to be able to compare to the single particle case, the nonadiabaticity is represented by the quantity $\sqrt{2\left(\left\langle x(T)^{2}\right\rangle-\epsilon_{x}(0)\right)} / A_{0}$, where $A_{0}$ is the amplitude of the driven oscillation, since this quantity applied to a single particle gives the same results as in Fig. 3. This nonadiabaticity is plotted versus the length of the ramp in Fig. 4. A similar behavior to the single particle case is observed. The major difference is the irregularity of the curve for the case with positive detuning that appears at the shorter ramps. This is due to the larger tune changes during the ramp since in these shorter ramps the step in the excitation amplitude is larger. Another

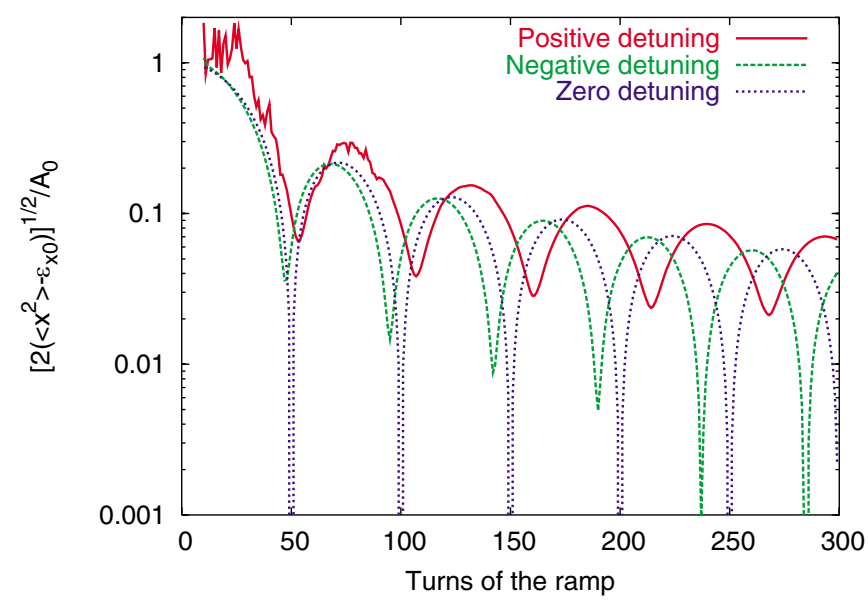

FIG. 4. (Color) Square root of twice the emittance blowup normalized to the amplitude of the driven oscillation with frequency $Q_{D}, A_{0}$ versus the turns of the ramp for negative, positive, and zero amplitude detuning. 
interesting feature is that an almost total adiabaticity is observed for two ramp lengths of the negative detuning curve. We conclude that the preferred nonlinear machine configurations, from the point of view of the adiabaticity, are (1) compensated amplitude detuning, and (2) an amplitude detuning that increases $Q_{-}$.

\section{THE LINEAR CASE WITH CHROMATICITY}

In the presence of chromaticity $Q_{x}^{\prime}$, a particle with momentum deviation $\varepsilon$ experiences a tune shift that depends on the turn number $T$ in the following way [13],

$$
\Delta Q_{x}(T)=\frac{Q_{x}^{\prime} \varepsilon}{2 \pi Q_{s} T}\left[\sin \left(2 \pi Q_{s} T+\phi_{0}\right)-\sin \left(\phi_{0}\right)\right],
$$

where $Q_{s}$ is the synchrotron tune and $\psi_{0}$ is the synchrotron phase at $T=0$. The turn-by-turn free motion of a particle is then expressed as

$$
\begin{aligned}
\hat{x}(T)-i \hat{p}_{x}(T) & =z e^{i 2 \pi\left(Q_{x}+\Delta Q_{x}(T)\right) T} \\
& =z e^{-i \xi \sin \phi_{0}} \sum_{q=-\infty}^{\infty} \mathrm{J}_{q}(\xi) e^{i 2 \pi\left(Q_{x}+q Q_{s}\right) T+q \phi_{0},}
\end{aligned}
$$

where $z$ contains the amplitude and phase of the transverse oscillation, $\xi$ is defined as $\xi=Q_{x}^{\prime} \varepsilon / Q_{s}$, and the exponential of the sine has been expanded as a series of Bessel functions. Each term of the series represents one synchrotron sideband. The same steps as in Sec. II will be given in order to describe the adiabaticity of the ramping process of an ac dipole with chromaticity. Therefore the first step is to derive an analytical solution of the particle motion with a constant ac dipole excitation in the presence of chromaticity. The coordinates at the exit of the ac dipole, $\hat{x}_{f}$ and $\hat{p}_{x f}$, are related to the coordinates at the entrance, $\hat{x}_{i}$ and $\hat{p}_{x i}$, at turn $n$ by the following expression,

$$
\hat{x}_{f}(n)-i \hat{p}_{x f}(n)=\hat{x}_{i}(n)-i \hat{p}_{x i}(n)+i k \cos \left(2 \pi Q_{D} n+\psi_{0}\right),
$$

where $k$ is the ac dipole excitation strength, $\sqrt{\beta_{D}} B L /\left(B_{0} \rho\right)$. If the ac dipole is connected at $n=0$, the particle coordinates at turn $T$ will be given by adding up all the ac kicks from the turns between 0 and $T-1$, propagated to the turn $T$. This propagation is realized by multiplying the ac kick by the exponential of the phase advance between turns $n$ and $T$ of the free chromatic oscillation. This phase advance is given by

$$
2 \pi\left(Q_{x}+\Delta Q_{x}(T)\right) T-2 \pi\left(Q_{x}+\Delta Q_{x}(n)\right) n .
$$

Therefore the particle coordinates at turn $T$ are given by

$$
\begin{aligned}
\hat{x}(T)-i \hat{p}_{x}(T)= & z e^{i 2 \pi\left(Q_{x}+\Delta Q_{x}(T)\right) T}+i k \sum_{n=0}^{T-1} \cos \left(2 \pi Q_{D} n\right. \\
& \left.+\psi_{0}\right) e^{i 2 \pi\left[\left(Q_{x}+\Delta Q_{x}(T)\right) T-\left(Q_{x}+\Delta Q_{x}(n)\right) n\right]} .
\end{aligned}
$$

To perform the summation over $n$ the cosine is expressed as the sum of two exponentials and the quantity $\exp \left(-i 2 \pi \Delta Q_{x}(n) n\right)$ is expanded in a series of Bessel functions, obtaining

$$
\begin{aligned}
\hat{x}(T)-i \hat{p}_{x}(T)= & z e^{i 2 \pi\left(Q_{x}+\Delta Q_{x}(T)\right) T}+\frac{i k}{2} e^{i \xi \sin \phi_{0}} \sum_{n=0, q=-\infty}^{T-1, \infty} \mathrm{J}_{q}(\xi) e^{i\left(2 \pi Q_{q-} n+\psi_{0}-q \phi_{0}\right)} e^{i 2 \pi\left(Q_{x}+\Delta Q_{x}(T)\right) T} \\
& +\frac{i k}{2} e^{i \xi \sin \phi_{0}} \sum_{n=0, q=-\infty}^{T-1, \infty} \mathrm{J}_{q}(\xi) e^{i\left(-2 \pi Q_{q+} n+\psi_{0}-q \phi_{0}\right)} e^{i 2 \pi\left(Q_{x}+\Delta Q_{x}(T)\right) T},
\end{aligned}
$$

where $Q_{q \pm}=Q_{D} \pm\left(Q_{x}+q Q_{s}\right)$. Performing the summation over $n$ gives

$$
\begin{aligned}
\hat{x}(T)-i \hat{p}_{x}(T)= & z e^{i 2 \pi\left(Q_{x}+\Delta Q_{x}(T)\right) T}+e^{i \xi \sin \phi_{0}} \sum_{q=-\infty}^{\infty} \delta_{q-} e^{i\left(2 \pi Q_{q-} T-q \phi_{0}\right)} e^{i 2 \pi\left(Q_{x}+\Delta Q_{x}(T)\right) T} \\
& -e^{i \xi \sin \phi_{0}} \sum_{q=-\infty}^{\infty} \delta_{q+} e^{i\left(-2 \pi Q_{q+} T-q \phi_{0}\right)} e^{i 2 \pi\left(Q_{x}+\Delta Q_{x}(T)\right) T}
\end{aligned}
$$

where

$$
\delta_{q \pm}=k \mathrm{~J}_{q}(\xi) \frac{e^{ \pm i\left(\pi Q_{q \pm}-\psi_{0}\right)}}{4 \sin \left(\pi Q_{q_{ \pm}}\right)}
$$

These expressions already describe the motion, but it is interesting to study the Fourier spectrum by performing the expansion of the remaining exponential of a sinus in terms of the Bessel functions, 


$$
\begin{aligned}
\hat{x}(T)-i \hat{p}_{x}(T)= & z e^{i 2 \pi\left(Q_{x}+\Delta Q_{x}(T)\right) T}+\sum_{q, p=-\infty}^{\infty} \delta_{q-} \mathrm{J}_{p}(\xi) e^{i\left[2 \pi\left(Q_{D}+(p-q) Q_{s}\right) T+(p-q) \phi_{0}\right]} \\
& -\sum_{q, p=-\infty}^{\infty} \delta_{q+} \mathrm{J}_{p}(\xi) e^{i\left[2 \pi\left(-Q_{D}+(p-q) Q_{s}\right) T+(p-q) \phi_{0}\right]} .
\end{aligned}
$$

The first term on the right-hand side of this equation represents the chromatic free oscillations. The second term contains an oscillation with the frequency of the ac dipole surrounded by an infinite set of sidebands with frequencies $Q_{D}+m Q_{s}$, where $m$ is any integer number. The third term is similar to the second but with the central frequency being the opposite of the ac dipole frequency. But this equation holds only for a particle with certain relative momentum deviation $\varepsilon$ and initial synchrotron phase $\phi_{0}$. The beam particles are distributed over the longitudinal plane, and in order to obtain the transverse motion it is mandatory to average over the longitudinal coordinates. The resulting transverse motion strongly depends on the initial longitudinal distribution. In particular, for a Gaussian beam centered and matched to the bucket no synchrotron sidebands are observed around the ac dipole frequencies. This is obtained by computing the following integral,

$$
\begin{aligned}
\int_{0}^{\infty} d \varepsilon \int_{0}^{2 \pi} d \phi_{0}\left(\hat{x}(T)-i \hat{p}_{x}(T)\right) \frac{\varepsilon}{2 \pi \sigma_{\varepsilon}^{2}} e^{-\varepsilon^{2} /\left(2 \sigma_{\varepsilon}^{2}\right)}= & e^{-s^{2}} \sum_{q=-\infty}^{\infty} \mathrm{I}_{q}\left(\mathrm{~s}^{2}\right)\left[z e^{i 2 \pi\left(Q_{x}+q Q_{s}\right) T}+\frac{k e^{-i\left(\pi Q_{q^{-}}-\psi_{0}\right)}}{4 \sin \left(\pi Q_{q^{-}}\right)} e^{i 2 \pi Q_{D} T}\right. \\
& \left.-\frac{k e^{i\left(\pi Q_{q_{+}}-\psi_{0}\right)}}{4 \sin \left(\pi Q_{q+}\right)} e^{-i 2 \pi Q_{D} T}\right],
\end{aligned}
$$

where $\varsigma=Q_{x}^{\prime} \sigma_{\varepsilon} / Q_{s}$. This equation describes the turn-by-turn motion of the bunch centroid in the presence of an ac dipole and chromaticity. Note that there are no sidebands around the frequencies $\pm Q_{D}$ as opposed to the single particle case in Eq. (24).

To describe the adiabaticity of the ramping process of an ac dipole with chromaticity the same procedure as in Sec. II is undertaken. Equation (21) is used to describe the single particle motion on the flattops of the steps of the excitation amplitude. The continuity equation of the transverse coordinates between the regions $n$ and $n+1$ is expressed as

$$
z^{(n+1)}=z^{(n)}+e^{i \xi \sin \phi_{0}} \sum_{q=-\infty}^{\infty} \delta_{q-} e^{i\left(2 \pi Q_{q^{-}} N(n+1)-q \phi_{0}\right)}-e^{i \xi \sin \phi_{0}} \sum_{q=-\infty}^{\infty} \delta_{q_{+}} e^{i\left(-2 \pi Q_{q^{+}} N(n+1)-q \phi_{0}\right)} .
$$

This equation is the analog to Eq. (6) and is solved in the same way. Taking $N=1$, the linear ramp, the amplitude and phase of the natural oscillation $z^{(n)}$, is given by

$$
z^{(n)}=z^{(0)}-e^{i \xi \sin \phi_{0}} \sum_{q^{\prime}=-\infty}^{\infty} \delta_{q^{-}} \frac{\sin \left(\pi Q_{q-} n\right)}{\sin \left(\pi Q_{q^{-}}\right)} e^{i\left(\pi Q_{q^{-}}(n+1)-q \phi_{0}\right)}+e^{i \xi \sin \phi_{0}} \sum_{q=-\infty}^{\infty} \delta_{q^{+}} \frac{\sin \left(\pi Q_{q+} n\right)}{\sin \left(\pi Q_{q^{+}}\right)} e^{i\left(-\pi Q_{q^{+}}(n+1)+q \phi_{0}\right)}
$$

To eliminate the dependence on the longitudinal coordinates the average over the longitudinal distribution has to be performed:

$$
\begin{aligned}
\int_{0}^{\infty} d \varepsilon \int_{0}^{2 \pi} d \phi_{0}\left(z^{(n)} e^{i 2 \pi(Q+\Delta Q(T)) T}\right) \frac{\varepsilon e^{-\varepsilon^{2} /\left(2 \sigma_{\varepsilon}^{2}\right)}}{2 \pi \sigma_{\varepsilon}^{2}}= & e^{-\varsigma^{2}} \sum_{q=-\infty}^{\infty} \mathrm{I}_{q}\left(\mathrm{~s}^{2}\right) e^{i 2 \pi\left(Q_{x}+q Q_{s}\right) T}\left[z^{(0)}+\frac{k^{(n)}}{n} \frac{\sin \left(\pi Q_{q-} n\right)}{4 \sin ^{2}\left(\pi Q_{q-}\right)} e^{i\left(\pi Q_{q^{-}} n+\psi_{0}\right)}\right. \\
& \left.-\frac{k^{(n)}}{n} \frac{\sin \left(\pi Q_{q+} n\right)}{4 \sin ^{2}\left(\pi Q_{q+}\right)} e^{i\left(-\pi Q_{q_{+}} n-\psi_{0}\right)}\right]
\end{aligned}
$$

where $k^{(n)}=\sqrt{\beta_{D}} B L^{(n)} /\left(B_{0} \rho\right)$. This expression provides the amplitude and phase of the natural tune and the synchrotron sidebands of the motion of the centroid in the flattop after a linear ramp of $n$ turns. From this expression it can be already concluded that a total adiabaticity cannot be achieved, even assuming $z^{(0)}=0$ and neglecting the $Q_{q+}$ term, since that would need a ramp of $P$ turns fulfilling

$$
P Q_{q^{-}}=j, \quad j \in N \quad \text { and } \quad \forall q .
$$

The nonadiabaticity is given by Eq. (2), where $|z|$ is given by the $z^{(n)}$ in Eq. (25). For the sake of simplicity, $z^{(0)}$ and the terms containing $Q_{q_{+}}$are neglected. The usual Gaussian bunch is assumed to perform the integral over the density distribution. The integral over the phase involves the computation of

$$
\frac{1}{2 \pi} \int_{0}^{2 \pi} d \phi_{0} z^{(n)} z^{(n)^{*}}=\sum_{q=-\infty}^{\infty}\left|\delta_{q^{-}}\right|^{2} \frac{\sin ^{2}\left(\pi Q_{q^{-}} n\right)}{\sin ^{2}\left(\pi Q_{q^{-}}\right)}
$$

The remaining integral over the energy is a tabulated one, finally giving 


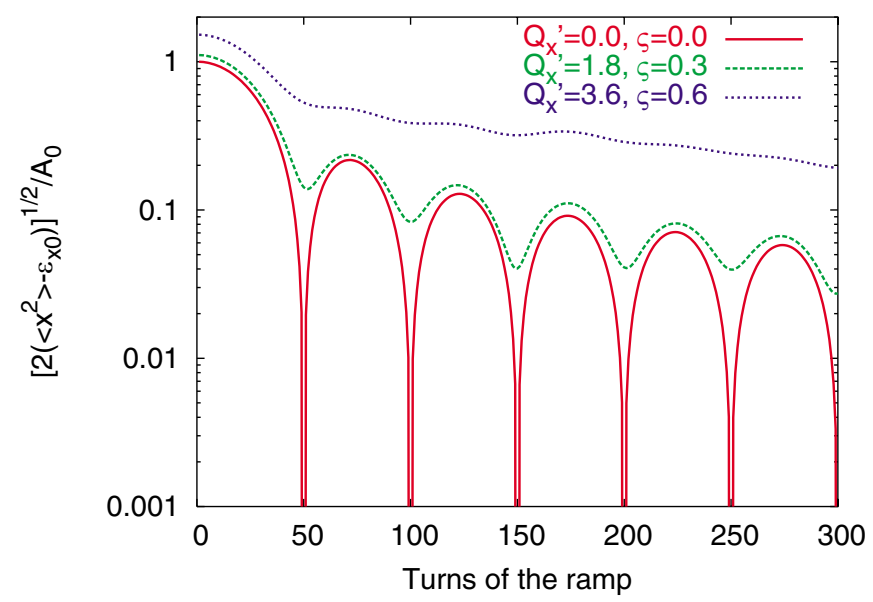

FIG. 5. (Color) Square root of twice the emittance blowup normalized to the amplitude of the driven oscillation with frequency $Q_{D}$, $A_{0}$ versus the turns of the ramp for three different values of the chromaticity.

$$
\left\langle x(T)^{2}\right\rangle-\epsilon_{x}(0)=\frac{k^{(n)^{2}}}{2 n^{2}} \sum_{q=-\infty}^{\infty} e^{-\varsigma^{2}} \mathrm{I}_{q}\left(\varsigma^{2}\right) \frac{\sin ^{2}\left(\pi Q_{q-} n\right)}{16 \sin ^{4}\left(\pi Q_{q-}\right)} .
$$

This equation gives the total nonadiabaticity of a linear ramp as a function of the number of turns and the chromaticity. Note that in the absence of chromaticity, $s=0$, this expression is in accordance with the results of Sec. II, mainly Eq. (9).

To illustrate the effect of chromaticity on the adiabaticity, Eq. (28) has been evaluated for different chromaticities. The CERN SPS parameters have been chosen, $Q s=$ $0.006, \sigma_{\varepsilon}=0.001, Q_{0-}=0.02$. In order to compare to Figs. 3 and 4 the quantity $\sqrt{2\left(\left\langle x(T)^{2}\right\rangle-\epsilon_{x}(0)\right)} / A_{0}$, where $A_{0}$ is the amplitude of the driven oscillation with frequency $Q_{D}$ as given by Eq. (26), is shown in Fig. 5 for three different values of the chromaticity, $Q_{x}^{\prime}=0.0,1.8,3.6$, or equivalently, $s=0.0,0.3,0.6$. Comparing the previous figures it is concluded that chromaticity dominates the deterioration of the adiabaticity of the ramp in machines like the CERN SPS. The same conclusion was achieved from experiments performed at the CERN SPS [10].

\section{MEASURING THE TUNE DURING THE RAMP}

This section aims to illustrate a way to measure the natural tune by computing the Fourier transform of the turn-by-turn centroid position during the ramp. A linear machine without chromaticity is considered in the following. From Eqs. (3) and (8) it is observed that the tune spectral line is excited during the ramp, although it may vanish completely at the flattop. From these equations it is possible to extract the Fourier component with the natural frequency $Q_{x}$ by expressing $\sin \left(\pi Q_{-} N n\right)$ as a sum of exponentials and taking $N=1$ (the linear ramp). This yields, for the Fourier component of frequency, $Q_{x}$,

$$
z^{(0)}+\frac{\delta_{-} e^{-i \pi Q_{-}}}{2 i \sin \left(\pi Q_{-}\right)}
$$

Assuming $z^{(0)}=0$, the amplitude of the natural tune spectral line from the data of a ramp of $n$ turns is given by

$$
\begin{aligned}
\left|\frac{\delta_{-}}{2 \sin \left(\pi Q_{-}\right)}\right| & =\left|\frac{\delta_{-}^{(n)}}{2 n \sin \left(\pi Q_{-}\right)}\right| \\
& =\frac{B L^{(n)}}{\left(B_{0} \rho\right)} \frac{\sqrt{\beta_{D}}}{8 n \sin ^{2}\left(\pi Q_{-}\right)}
\end{aligned}
$$

To perform the measurement in a real machine this amplitude needs to be larger than the noise of the turn-by-turn orbit measurement. Another important constraint is that the frequency resolution of the Fourier transform must be smaller than $Q_{-}$in order to resolve the two peaks with frequencies $Q_{x}$ and $Q_{D}$. The use of more accurate algorithms than the fast Fourier transform to obtain the Fourier spectrum seems to be needed when tight constraints are imposed [14,15]. Therefore a compromise among the quantities $n, \delta^{(n)}$, and $Q_{-}$should be achieved in order to obtain the required accuracy in the tune measurement.

To illustrate this kind of measurement a simulation has been performed using the same model as above. The following ac dipole parameters have been chosen: $n=$ $1100, \delta^{(n)}=9.6 \mathrm{~mm}$, and $Q_{-}=0.02$. From Eq. (30) this configuration yields a natural tune amplitude of $70 \mu \mathrm{m}$ during the ramp. The Fourier transform of the simulated turn-by-turn position during the ramping process is shown in Fig. 6. The natural tune amplitude shown in the plot is in perfect agreement with the prediction from Eq. (30).

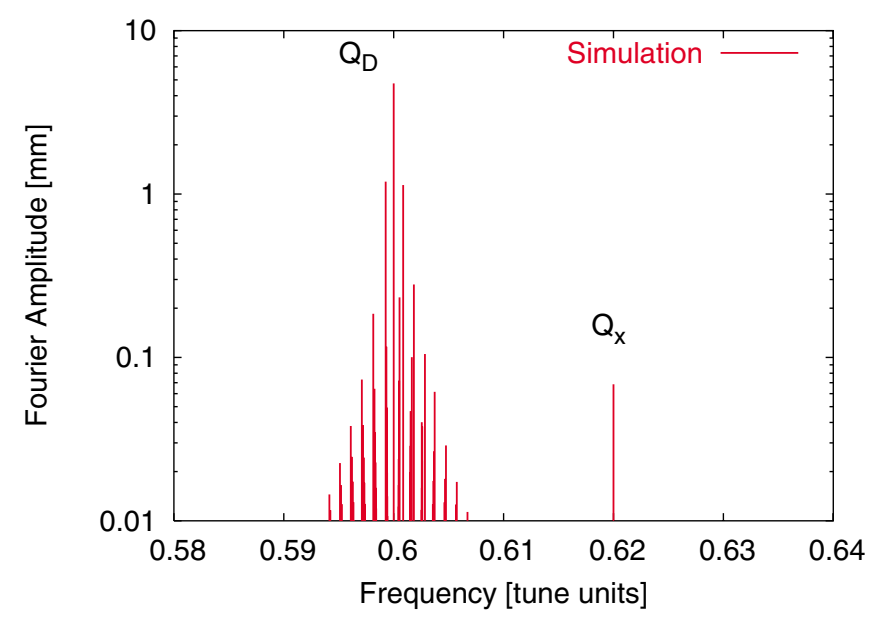

FIG. 6. (Color) Fourier spectrum of the simulated turn-by-turn particle position during the ramping process of an ac dipole. The natural and the ac dipole tunes are labeled with $Q_{x}$ and $Q_{D}$, respectively. 


\section{CONCLUSION}

Analytical expressions quantifying the adiabaticity of an ac dipole ramp have been achieved for a linear machine and for a machine with chromaticity modeled by a phase space rotation. It has been demonstrated that a nonlinear machine with compensated amplitude detuning is equivalent to a linear machine from the point of view of the adiabaticity of the ramping process. From the simulations and the analytical expressions it is concluded that, for machines like the CERN SPS, chromaticity dominates the deterioration of the ramping process of an ac dipole, in accordance with experimental observations [10]. Lastly, a nondestructive way to measure the natural tune by computing the Fourier spectrum of the turn-by-turn data acquired during the ramp is described.

\section{ACKNOWLEDGMENTS}

I thank M. Bai, W. Fisher, S. Peggs, and T. Roser for fruitful and motivating discussions. This work has been performed under the auspices of the United States Department of Energy.

[1] M. Bai, S. Y. Lee, J. W. Glenn, H. Huang, L. Ratner, T. Roser, M. J. Syphers, and W. van Asselt, Phys. Rev. E 56, 6002 (1997).

[2] M. Bai, L. Ahrens, J. Alessi, K. Brown, G. Bunce, P. Cameron, C. M. Chu, J.W. Glenn, H. Huang, A.E. Kponou, K. Krueger, W. Lamble, A. Luccio, Y.I. Makdisi, S. Y. Lee, M. Okamura, L. Ratner, K. Reece, T. Roser, H. Spinka, M. J. Syphers, N. Tsoupas, D. G.
Underwood, W. van Asselt, N. Williams, and A. Yokosawa, Phys. Rev. Lett. 80, 4673 (1998).

[3] S. Peggs and C. Tang, BNL Report No. RHIC/AP/159, 1998

[4] R. Tomás, Phys. Rev. ST Accel. Beams 5, 54001 (2002).

[5] M. Bai, S. Peggs, T. Roser, T. Satogata, and D. Trbojevic, inProceedings of the 2003 Particle Accelerator Conference, Portland, OR (IEEE, Piscataway, NJ, 2003).

[6] M. Bai, F. Pilat, T. Satogata, F. Schmidt, and R. Tomás, in Proceedings of the 2003 Particle Accelerator Conference, Portland, OR (Ref. [5]).

[7] S. Fartoukh, N. Catalan-Lasheras, and J. P. Koutchouk, in Proceedings of the 2003 Particle Accelerator Conference, Portland, OR (Ref. [5]).

[8] F. Schmidt, R. Tomás, N. Catalán, G. Crockford, S. Fartoukh, M. Hayes, W. Hofle, and J-P. Koutchouk, CERN Report No. AB-Note-2003-031 MD, 2003.

[9] R. Tomás, M. Bai, W. Fischer, A. Franchi, and G. Rumolo, in Proceedings of the 2004 European Particle Accelerator Conference, Lucerne, Switzerland (IEEE, Piscataway, NJ, 2004).

[10] O. Berrig, W. Hofle, R. Jones, J. Koopman, J.P. Koutchouk, and F. Schmidt, CERN Report No. SL-Note2000-062-MD, 2001.

[11] G. Arduini, R. Tomas, F. Zimmermann, and A. FausGolfe, in Proceedings of the 2003 Particle Accelerator Conference, Portland, OR (Ref. [5]).

[12] F. Schmidt, CERN Report No. SL/94-56 (AP).

[13] R. E. Meller, A. W. Chao, J. M. Peterson, S. G. Peggs, and M. Furman, Superconducting Super Collider Laboratory Report No. SSC-N-360, 1987.

[14] J. Laskar, C. Froeschlé, and A. Celletti, Physica (Amsterdam) 56D, 253 (1992).

[15] R. Bartolini, A. Bazzani, M. Giovannozzi, W. Scandale, and E. Todesco, Part. Accel. 56, 167 (1996). 